\title{
Erratum to: Discrete Lie Advection of Differential Forms
}

\author{
P. Mullen • A. McKenzie - D. Pavlov • L. Durant • \\ Y. Tong • E. Kanso · J.E. Marsden • M. Desbrun
}

Published online: 12 February 2011

(C) SFoCM 2011

\section{Erratum to: Found Comput Math \\ DOI 10.1007/s10208-010-9076-y}

This article did not contain the received/revised/accepted dates upon online publication. They are:

Received: 10/10/2008; Revised: 7/16/10; Accepted: 8/10/10

We regret the error.

The online version of the original article can be found under doi:10.1007/s10208-010-9076-y.

P. Mullen · A. McKenzie · D. Pavlov · L. Durant · J.E. Marsden · M. Desbrun ( $\varangle)$

Computing + Mathematical Sciences, California Institute of Technology, Pasadena, CA 91125, USA

e-mail: mathieu@cs.caltech.edu

\section{Y. Tong}

Computer Science \& Engineering, Michigan State University, East Lansing, MI 48824, USA

E. Kanso

Aerospace and Mechanical Engineering, University of Southern California, Los Angeles, CA 90089, USA 